\title{
De Leibniz a la arquitectura
}

\section{From Leibniz to architecture}

\author{
Maritza Granados Manjarrés \\ Departamento de Estética de la Facultad de Arquitectura y Diseño, Pontificia \\ Universidad Javeriana. Bogotá, Colombia. \\ granados.m@javeriana.edu.co
}

\section{Resumen}

El artículo busca hacer un traslado de la filosofía leibniziana al mundo de la arquitectura a través de la ley de la continuidad, planteando una serie de cinco leyes o condiciones que posibilitan dicho traslado y que a su vez fundan una teoría sobre cómo puede ser entendido el espacio arquitectónico a la luz de la filosofía. Además de la continuidad, el artículo aborda el problema del pliegue y su condición de dispositivo que puede ser entendido como el detonante primordial del espacio y la experimentación formal.

Palabras clave: Leibniz, arquitectura, superficie, pliegue.

\section{Abstract}

This paper tries to make a transit from the Leibnizian philosophy to the world of architecture through his Law of Continuity. This transit proposes five laws or conditions inherent to the architectonic space which establishes a theory on how space can be understood from a discipline like philosophy. Besides this Law, the paper addresses the problem of the fold and how it is understood as a device and the fundamental trigger of space and formal experimentation.

Keywords: Leibniz, Architecture, Surface, Fold. 


\title{
Introducción
}

\begin{abstract}
Al hacer un balance, ¿qué es un pliegue? Un germen de forma. Pero, ¿qué es un germen sino un conjunto de pliegues? El pliegue es el elemento de la forma, el átomo de la forma, sí, su clinamen.

Pero, ¿qué es una forma? Respuesta: algo liso con pliegues.
\end{abstract}

M. Serres

Supongamos que el espacio es infinito, pero no cualquier espacio: el nuestro, el arquitectónico. Ahora, a esa infinitud sumémosle flexibilidad absoluta. Pensémoslo como una superficie flexible y elástica que puede ser modificada tantas veces como se quiera, sin que se agote, y sin que se pierdan sus propiedades topológicas. Es en estas modificaciones donde se generan nuevas espacialidades, nuevas habitaciones y relaciones con otros espacios. Pero si esta superficie es infinita y flexible significa que debe ser continua, sin vacíos, ni saltos. Así que pensemos en continuidad, mejor, pensemos en la ley de continuidad.

Alrededor de 1680, Gottfried Leibniz, filósofo y matemático alemán, planteaba una serie de condiciones que dieron lugar a lo que se convirtió en la ley de la continuidad cuya teoría intentará ser extrapolada al mundo de la arquitectura a partir de distintos autores, pero particularmente la tesis doctoral de Manuel Luna Alcoba (1994), titulada "La ley de la continuidad en G. W. Leibniz", destaca en por su detallado esfuerzo. . Ahora bien, esta extrapolación tiene una intención formal: de la especulación que resulta de entender la ley de la continuidad y, por consiguiente, la aparición del pliegue, habrá un producto que tome forma y constituya la materialización resultante del ejercicio de trasladar la filosofía al mundo de la arquitectura.

\section{Antecedentes: pliegue, continuidad y espacio}

La continuidad aquí propuesta se entiende en términos de pliegue. Leibniz la define como "la línea que constituye el ángulo en que se curvan dos superficies continuas" (Luna 100) y sostiene que el continuo sigue una estructura de pliegue, al cual contrapone el concepto de despliegue -en tanto proceso de análisis, actualización o descomposición-, afirmando que cuando sucede no se anula el pliegue, más bien se multiplica para darle nuevas formas al plegamiento. Es decir, siempre que se quiera llevar a cabo un proceso de análisis aparecerán nuevos pliegues, con la acción de explicar, de desplegar. Incluso, si se rastrea el origen de la palabra plegar ${ }^{1}$, fácilmente se

1 Según el diccionario etimológico de Monlau: “Plegar. Plicare: en g. plekein, plegar, doblar, juntar entrelazar, hacer 
advierte que sus derivados incluyen términos como duplicar e implicar, que al igual que el despliegue dan paso e incluso obligan la aparición de nuevos plegamientos como solución a los esfuerzos que surgen del desplegar. Dicho de otro modo, a cada pliegue le corresponde una multiplicidad de ellos mismos y cada uno está provisto de inflexiones, entendidas como los puntos de variación. Esto quiere decir al menos dos cosas: primero, que las relaciones entre superficies se constituyen con el pliegue, y a su vez este origina singularidades. En otras palabras, la superficie continua se ve afectada por el pliegue al incorporar atributos en ella, o a la inversa, que las singularidades fundan esos pliegues. Segundo, que dicha multiplicidad de singularidades conforman lo real. Por lo tanto, solamente hay continuidad si existe un pliegue, "un giro material que descompone la secuencia y que -a la vez-, la hace existir en tanto que línea de variabilidad" (Colodro 62), que es lo mismo que proponer que el pliegue singulariza la secuencia, la vuelve cualitativa.

Sin embargo, aun con estas singularidades es propio del continuo que los elementos que lo componen no sean totalmente distintos, es decir, que exista cierta homogeneidad y orden, una relación a pesar de sus diferencias. En otras palabras, a partir de dicho orden aparece la continuidad, se garantiza, al punto de la reciprocidad, es decir, hay orden porque hay continuidad y continuidad porque hay orden. Esto significa que al cumplir con estas dos características las partes tienen algo en común y eso las hace continuas, a diferencias de aquellas que, aunque se toquen o yuxtapongan, solo resultan ser contiguas.

Ahora, con las implicancias que suponen las propiedades que definen el continuo, Leibniz propone anticipadamente uno de los principios que más tarde será clave en la teoría de la evolución: Natura non facit saltus, cuya traducción literal es la "naturaleza no da saltos". Tal afirmación se convierte después en el pilar de la ley de la continuidad que arguye que en la naturaleza no existen vacíos, aun cuando más adelante -con el advenimiento de la mecánica cuántica- los saltos cuánticos lo contradijeran. Sin embargo, lo interesante de esta afirmación es poder entender que, en ciertos fenómenos, nada se hace repentinamente, sino que siempre hay un tránsito entre las partes - de un lado a otro-, pasando por sus puntos medios, es decir, un tránsito ordenado, como el existente de una línea a la superficie o en los cambios de velocidad. Uno de estos fenómenos es la aparición del espacio arquitectónico y el espacio habitable. De este modo, la reflexión desarrollada aquí no solo pretende realizar un traslado de la filosofía leibniziana al espacio arquitectónico a partir de estas nociones de pliegue, continuidad, orden y homogeneidad, sino que propone el espacio construido y por construir como una manifestación que vale la pena ser pensada desde la ley de la continuidad.

dobleces o pliegues, etc. Aplicar y sus derivados [...] Complicar, Cómplice, Duplicar, Explicar, Explicito, Explotar, Implicar, Implícito, Multiplicar, Simple (de sine plexu, o sine plicis, sin doblez, sin pliegue) Súplica, Suplicar" (375). 
Es así como aparece el pentálogo que recoge las leyes que regulan y proponen el espacio arquitectónico como continuo:

\section{De Leibniz al espacio arquitectónico - pentálogo}

El espacio nace del lugar.

La continuidad es una propiedad del espacio.

Son determinantes de la continuidad la homogeneidad y el orden.

No hay tránsito por saltos, siempre se pasa por puntos intermedios.

El continuo sigue una estructura de pliegue.

\section{El espacio nace del lugar}

Para Leibniz, el espacio es una extensión sin cambios y sin límites (Luna 192) y, además, en la extensión se mide el espacio. Ambas cosas, extensión y espacio, están compuestas o nacen de instancias menores. Por un lado, la extensión nace por el situs, el cual, aunque no se refiere al lugar, constituye un espacio en potencia siempre que se convierta en un continuo. Junto con ello, se diferencia como punto en el espacio a través del positio que convierte diversos situs en una serie continua. Por otro lado, el espacio nace del lugar, o más bien, de una difusión de este, que está compuesto de puntos o lugares más simples, es decir, podría entenderse el espacio como una sumatoria de lugares donde cada uno de esos lugares ocupa un único espacio que no puede compartirse ni igualarse con otro (fig. 1).

A la luz de estas afirmaciones aparece además una de las conclusiones más interesantes y sugerentes que realiza Luna: la aparición de los espacios existentes abre todo un universo sobre los espacios potenciales que habitan el mundo construido cuando se traslada esta ley al espacio arquitectónico: "Si efectivamente el espacio es definido en función de los coexistentes, Leibniz podía haber propuesto la existencia de una pluralidad de espacios posibles tantos como mundos, de los cuales sólo uno, el mejor, existiría realmente" (Luna 202).

Queda claro entonces que esta relación extensión/espacio (fig. 2) es indisoluble y además recíproca, pues la extensión queda establecida cuando aparece el espacio y se convierte en magnitud de esta, y por su parte el espacio establece magnitudes en la extensión cuando aparece. De este modo, tal y como concluye Luna, la continuidad es una condición intrínseca del espacio, es decir, está sujeta a la ley de la continuidad y se enmarca en un sistema ordenado y homogéneo.

Pero volvamos al espacio arquitectónico. Lugar es uno de los términos más analizados en arquitectura por lo que implica para el usuario, muy cercano a ese lugar antropológico propuesto por Augé en el que se trabaja, se vive y también se defiende y se cuida: "un lugar puede definirse como lugar de identidad, relacional e histórico" (Augé 83). Además, es el lugar el cual se consagra a través de la arquitectura, todo un 
FIGURA 1

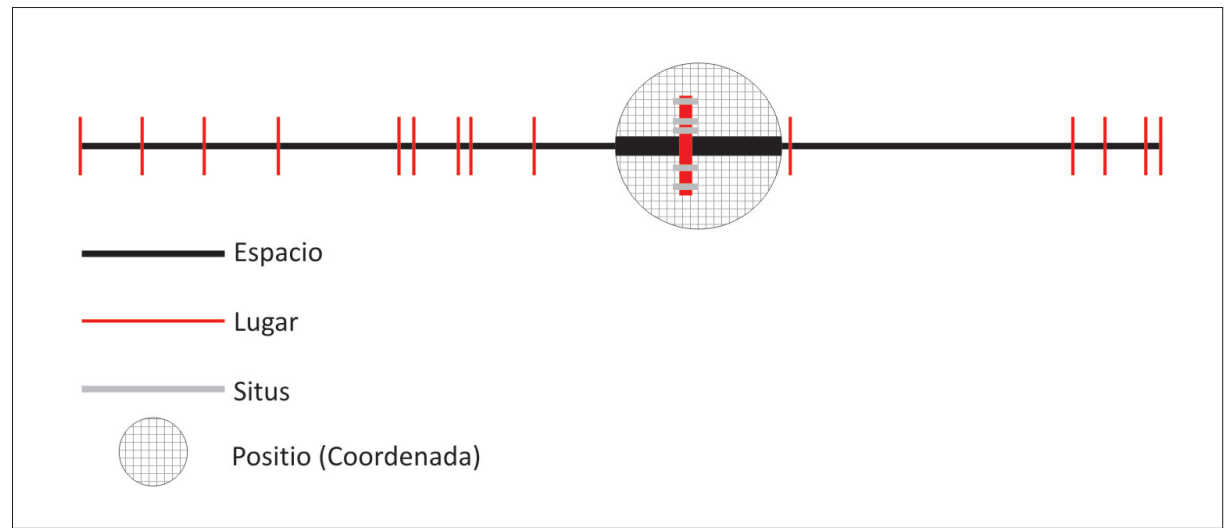

Esquema de espacio, lugar y situs.

\section{FIGURA 2}

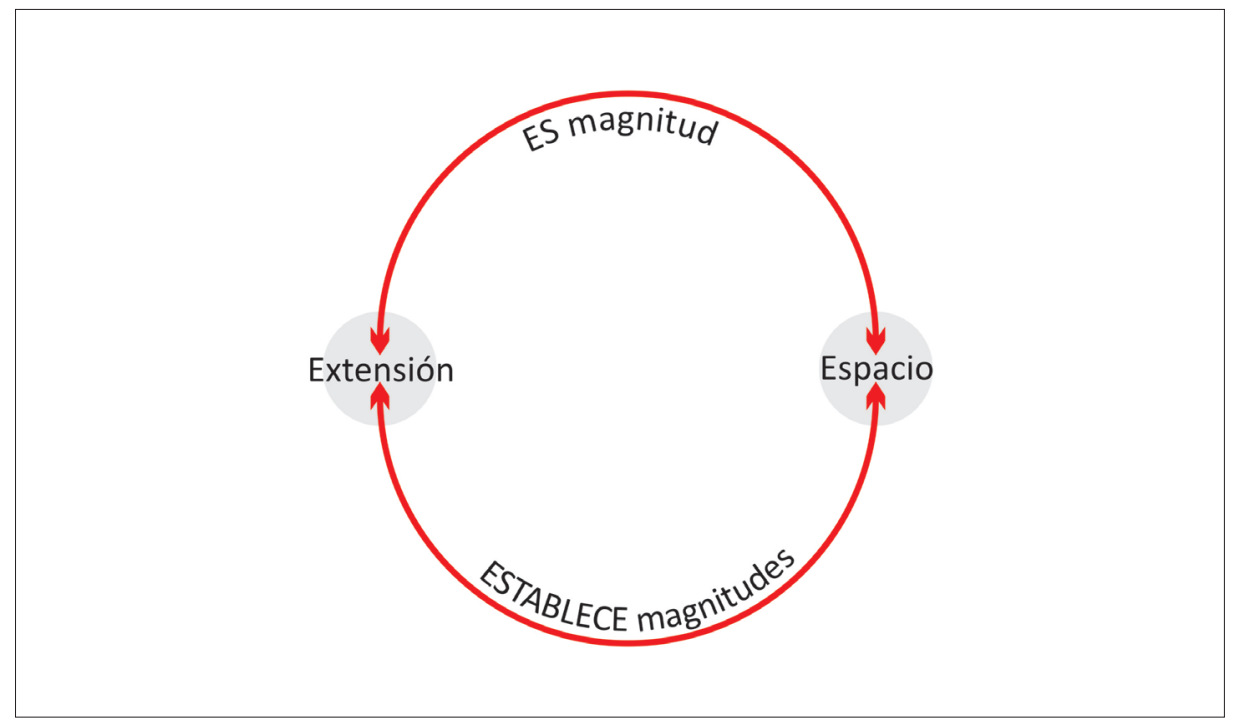

Esquema de reciprocidad extensión/espacio.

dispositivo que permite que el lugar se constituya como tal junto con las prácticas sociales que lo habitan.

Ahora, si para Leibniz el espacio surge del lugar y no al contrario -como se propone en arquitectura- esto supone no solo una contradicción sino, lo que es mejor, propone todo un lugar de análisis. Si es cierto que el espacio existe en tanto haya lugares que lo definan, y no solo los antropológicos de Augé, sino también aquellos únicos de Leibniz, ¿cómo se definiría el espacio arquitectónico? Pues, 
siguiendo a Leibniz, es interesante pensar que el espacio arquitectónico solo pueda definirse como una sumatoria de lugares que, en su condición de singulares y únicos, al ser habitados otorguen al espacio esa cualidad de representantes de la identidad, de modo que solo pueda haber espacio arquitectónico siempre que haya un lugar que lo contenga.

\section{La continuidad es una propiedad del espacio}

El espacio se reconoce como continuo incluso antes de la aparición de la ley de la continuidad. De hecho, el problema sobre la extensión surge de pensar el espacio como continuo. Pero entonces la cuestión es ¿cómo se piensa la continuidad en el espacio arquitectónico? Para Leibniz: “Continuo es un todo cuyas partes están - una fuera de otra e- indeterminadas [...] Verdaderamente las partes de continuo son indeterminadas porque no están ya asignadas, sino que pueden designarse a voluntad, así se distingue de lo contiguo" (cit. en Luna 94). De modo que que las partes del continuo solo aparecen cuando son resueltas, y en esa resolución se diferencian completamente de otras, aun cuando son de una misma naturaleza.

Desde esta continuidad se propone entender el espacio arquitectónico en tanto un espacio continuo que se funda sobre una única superficie que puede ser infinitamente modificable sin perder sus propiedades, y dichas modificaciones pueden ser de orden material o pueden darse a partir del acto mismo de habitabilidad. Es decir, el objeto arquitectónico surge de una única superficie que en su flexibilidad configura un espacio habitable. Por lo tanto, la resolución del continuo arquitectónico reside en el acto creativo del ejercicio proyectual o de la habitabilidad. Precisamente, es cuando surge esta resolución que el espacio deja de ser indeterminado: se fragua como arquitectónico y allí se funda la diferenciación entre distintas arquitecturas aun cuando pertenecen al mismo universo de creación. Es en esta pertenencia donde se modela la tercera ley.

\section{Son determinantes de la continuidad la homogeneidad y el orden}

Para Leibniz es claro que orden y homogeneidad son requisitos para que exista el continuo, y para que el espacio sea continuo debiera gozar de dichas características. Pero, ¿ cómo se piensa esto en términos de arquitectura? En principio, pensar el espacio como ordenado y homogéneo puede resultar difícil si nos apegamos al imaginario que se tiene de esto, donde todo es igual y se estructura con tanta similitud que es difícil diferenciar; sin embargo, aquí estriba una de las propuestas más contundentes de Leibniz sobre continuo, la cual resulta aplicable al espacio arquitectónico: 
Es la semejanza la que otorga universalidad a la homogeneidad, la que permite pasar de una cosa a otra. De hecho, pueden definirse lo homogéneos como aquellos que, o son semejantes, o pueden hacerse similares por una transformación. Pero la homogeneidad no sería tal si esa semejanza no estuviese compensada o regulada con la igualdad. Para decirlo de un modo más simple, son homogéneos los que participan de una naturaleza común (cit. en Luna 89).

De allí que, por un lado, se piensa en espacios que son homogéneos en la medida en que todos participan de las mimas propiedades o condiciones. Pensemos en la ciudad y en que está desarrollada sobre esa superficie flexible e infinita que se propone. En esa ciudad se pueden diferenciar todas sus intervenciones, no obstante, es leída como homogénea, en la medida en que todas las intervenciones pertenecen al mismo universo de lo creado. Casi cualquier nivel de espacio puede imaginarse de este modo. Ya no consideremos la ciudad, sino nuestra casa, en el espacio en el que estamos ahora, el que compartimos con el resto de espacios que hemos conocido antes, su homogeneidad en términos de su naturaleza aun cuando se diferencien enormemente unos de otros.

Por otro lado, está el orden, el cual es muchísimo más fácil de pensar y se propone como "la relación entre muchos que se diferencian entre sí" (Luna 90). Entonces, todo el espacio arquitectónico efectivamente participa de una naturaleza común y eso lo hace homogéneo, y claro, en la medida en que dicho espacio está relacionado hay orden, y también continuidad. Vaya a la ciudad nuevamente, su experiencia en ella, la manera en que la recorre diariamente y, en efecto, la sentirá como un continuo que aun cuando está mediado por la velocidad, no pierde esta condición; claro está que la naturaleza de la ciudad es la misma. Homogeneidad no indica necesariamente igualdad, solamente indica que comparten atributos o propiedades. Ahora, estos vínculos que se fundan en el continuo no solamente son espaciales, son también sociales, relacionales, incluso nuestras relaciones pueden ser pensadas como homogéneas y continuas.

Oriol Nel.lo, geógrafo barcelonés especialista en estudios urbanos y ordenación del territorio define la ciudad mo una red que debe ser continua y tales continuos: son hoy definidos por redes de relaciones, por la telaraña de relaciones que el movimiento de personas, mercancías y de información tejen sobre el territorio. La ciudad actual es, pues, un red. Ahora bien, cada actividad, cada tipo de relación, teje su propia red, que se superpone a las demás. La ciudad es pues una red de redes. Pero la extensión y la intensidad de estas rede varía en diversos momentos del día, de la semana, o del año: son de geometría variable. La ciudad, pues, es una red de redes de geometría variable (cit. en Centelles Portella 73).

Así que nos movemos sobre una red de redes, red de espacio y red de relaciones, casi fractales, que crecen y decrecen constantemente; sin embargo, mediadas por dichas características dan además lugar a la aparición del pliegue. 


\section{No hay tránsito por saltos, siempre se pasa por puntos intermedios}

Una de las grandes máximas de Leibniz, que fundamenta la ley de la continuidad tiene que ver con que nada se hace en un solo tiempo: se requieren todos los intermedios. Es decir existe un tránsito que siempre necesita de las instancias anteriores e intermedias para que este suceda. Como señala Luna: "implica que se pasa siempre de lo grande a lo pequeño y viceversa por lo mediano, en los grados como en las partes, y que nunca un movimiento nace inmediatamente del reposo ni vuelve a él si no es por un movimiento más pequeño" (128). Por lo tanto, para que la continuidad se mantenga no deben existir vacíos porque allí se vería interrumpida, se acabaría con ella. En el sistema leibniziano, esta continuidad, no rige solamente el movimiento o la naturaleza, también es aplicable a las matemáticas, física, psicología u ontología, y naturalmente a lo monadológico donde no se pasa de una mónada a otra sino por medio de su transformación (Bueno Martínez 149). Entonces si el espacio, arquitectónico y no, forma parte de ese sistema, cualquier cambio o movimiento en su interior dependerá de un tránsito y no podrá hacerse por saltos. Esto también aplica para el espacio construido.

La experiencia en el espacio siempre será continua: no hay manera de que uno vaya de un lugar a otro sin tener la experiencia de aquellos intermedios. El problema es que a veces dicha experiencia parece no ser gratificante, incluso que se pierda en la rutina. Es posible que uno no perciba dicha continuidad por un bloqueo en la percepción que, en todo caso, está en manos de los arquitectos solucionar². La propuesta es pensar que en tal continuidad sin saltos, todo aquello que en términos espaciales a venga a nuestro encuentro puede ser pensado como pliegue, como una inflexión en una superficie modificable.

\section{El continuo sigue una estructura de pliegue}

A propósito de la división en partes, Leibniz propone que estas no deben ser pensadas como un último resultado, al contrario, siempre que se requiera más análisis dichas partes podrán posteriormente dividirse. De hecho, Leibniz propone que la división del continuo debe ser pensada desde lo que ocurre en un papel cuando es plegado: aun cuando dicho plegamiento sea infinito la superficie no se disocia ni se separa (Luna 99).

2 A propósito del fenómeno de la calidad de las nuevas arquitecturas y la experiencia escribí el artículo “OOcupar o habitar? Aproximación al fenómeno actual" (376-91) en la revista Arte, Individuo y Sociedad. 
Así, a la luz de continuidad y pliegue en tanto herramientas de observación, análisis y descomposición, puede pensarse la noción de espacialidad. Es decir, para que exista el pliegue debe existir una superficie, y dicha superficie debe ser continua:

La gracia del pliegue es que ocurre sobre la superficie compartida y tiene la habilidad de manipular/unir elementos. El pliegue anima la superficie relacionando elementos sobre un plano continuo y al mismo tiempo diferenciado la superficie (Oleastro 111).

Uno de los grupos de mayor interés en la vanguardia española de los años cincuenta fue el grupo cordobés Equipo 57, fundado en 1957 después de una exposición en el Café Rond Point de París donde empezó la presentación de un conjunto de manifiestos que cimentarían su quehacer artístico mediante una serie de planteamientos que llamaban a la interdisciplinariedad y a la globalidad de intereses. Ese mismo año, publican un manifiesto estético conocido como Interactividad del Espacio Plástico. Si bien en su momento "denunciaba el mercantilismo del arte y el estancamiento de las formasplásticas y se propugnaba un método de trabajo colectivo, sin personalismos ni autoría individual" (Centro Andaluz de Arte Contemporáneo), interesa la concepción que ellos tenían de la materia y el espacio. En abril de 1958, a propósito de una exposición en el Museo Thorvaldsen en Copenhague, escriben en su catálogo:

la continuidad dinámica se produce por la comunicación de las concavidades y de las convexidades a través de una zona de inflexión. La inflexión es siempre cóncavo-convexa en cualquiera de los espacios que se consideren. Por la inflexión ambos espacios ocupan todos los términos posibles: el espacio continente pasa a ser espacio contenido, y a la inversa, produciéndose la unidad [...] en la arquitectura se plantea un solo espacio y una sola superficie límite, que distribuye la acción de este espacio. Esta superficie en sí continua, por su doble curvatura cóncavo-convexa disipa el espacio en todas direcciones. La continuidad activa del espacio implica la abolición de la superficie plana o curva en una sola dirección (cit. en Ureña 164-5)

La propuesta del espacio como elemento disipativo abre la posibilidad de concebirlo como un elemento que se desliza sobre las superficies configurándolas y deformándolas con el fin de generar nuevas espacialidades. A su vez, plantea la pregunta sobre el rol que juega el pliegue en la conformación de dicha espacialidad, es decir, no solo en tanto elemento que promueve su continuidad -como se vio anteriormente-, sino también como un elemento que lo califica, porque dentro de esa inflexión cóncavoconvexa el espacio es diferenciado, a pesar de su condición de continuo.

A la luz de dicha diferenciación, el espacio podría situarse dentro o fuera de esta inflexión o pliegue, pero, en cualquiera de los dos casos, como propone Pérgolis a partir de Serres, el pliegue es el lugar: "define un en, una preposición, un operador que 
indica las relaciones de posición, el estar ahí y sus relaciones con el exterior" (49). Por lo tanto, si el espacio se ubica dentro o fuera del pliegue -es decir, si es contenido o no por las superficies que devienen con el pliegue- simplemente califica como interno o externo a él, pero sigue configurando lugar. Tal como se propone con el Espacio Plástico, este puede ser a su vez contenido o continente, al deslizarse continuamente sobre la superficie plegada.

En el infinito espacio disponible para ser configurado, así como en la superficie donde reposa, el pliegue otorga singularidad al espacio, la característica de disiparlo o contenerlo, pero siempre proponiéndolo como lugar que se confirma, concreta y fragua, al habitarlo.

Junto con ello, Leibniz propone que un pliegue está compuesto por varios de ellos mismos que surgen en los procesos de análisis. En este sentido, la estructura de pliegue puede analizarse tan profundamente como se desee, implicando un acto de despliegue que, lejos de disociar la superficie o anular el pliegue, evidencia un conjunto de otros. Pensémoslo de esta manera, si nos situamos en el espacio continuo y comprendemos el pliegue como la línea que singulariza las superficies, emerge la posibilidad de abordar el pliegue no solo desde la teoría como propone Leibniz, o en una superficie real como el gesto que la singulariza sino que puede entenderse como un evento.

Una vez más, situémonos en el espacio arquitectónico, hagamos el ejercicio de trasladar al pliegue todos los accidentes que modifican el espacio, pero no solo los que existen, sino también los probables, aquellos que en potencia pudieron haber existido. Cada uno de estas variaciones o eventos están compuestos de una serie de ellos mismos. En definitiva, el espacio se configura como su sumatoria de lo que es, pero también es lo que podría ser.

En la teoría de la probabilidad, que mide sus resultados dependiendo de la posibilidad de que estos ocurran, existen una serie de fenómenos que se consideran experimentos aleatorios cuyo resultado no puede predecirse con exactitud, aunque si especificarse cuáles podrían ser. Todos esos resultados posibles, conocidos como $\Omega$, crean un conjunto denominado espacio muestral, que es discreto si el conjunto de resultados es finito, y continuo si es infinito no numerable, es decir: " si el conjunto anterior está formado por intervalos continuos, de forma que entre dos resultados cualesquiera existen infinitos resultados posibles" (Cáceres Hernández 149). De este modo se comporta el ejercicio proyectual: donde hay infinitos resultados posibles y todos en potencia configuran el lugar. Por lo tanto, si quisiéramos hacer un ejercicio de análisis de una arquitectura específica y lo desplegáramos junto con el lugar donde se implanta entonces aparecerían todas aquellas inflexiones que son leídas como otros pliegues sobre uno ya existente; de hecho, aquel espacio no existiría si no fuera por esos otros que por ahora son potencia. 


\title{
¿Y la mónada?
}

La mónada es tanto la causa del espacio como de todo lo demás: el lugar de creación, el punto desde el cual todo surge. Según Bueno Martínez, es en los fenómenos biológicos donde se encuentra más relación con toda la teoría monadológica de Leibniz, al punto que es posible comparar una célula con una mónada, debido a que la primera es considerada una unidad viviente, dotada de un alma, concebida como una estructura diminuta que compone la vida. Esta noción de célula se extendió para comprender los vegetales y animales. Lo interesante de ello es que concibe los organismos en tanto compuestos de varias unidades o mónadas vivientes que gozan de una armonía entre sí y forman un sistema. Estas reflexiones lo llevan a reflexionar sobre cómo transitarse desde la biología y al espacio, llegando a suponer que los fenómenos geométricos fueron pensados en término de los biológicos (Bueno Martínez 37-44). Además, apunta lo siguiente:

\begin{abstract}
¿Cuál es el concepto geométrico más próximo a la Idea de Mónada, el equivalente geométrico de la persona a la teología, de la célula a la biología, o del elemento químico (átomo) en la física? Sin duda el concepto de punto. Porque el punto geométrico, tal como Leibniz lo trata, reproduce de algún modo, en el Espacio, la estructura de la Mónada, sí como la continuidad entre los puntos [...] refleja en el ámbito del espacio, el gradualismo que rige las relaciones entre las infinitas mónadas reales. A fin de cuentas (podríamos decir por nuestra parte) si bien el espacio (a cuya categoría pertenece el punto geométrico) es la negación de la unidad (partes extrapartes) el punto es, a su vez, la negación espacial del espacio, su límite interno: en ese límite podríamos reencontrarnos con la idea de mónada. Pues el punto es ya inextenso, sin partes, como la mónada: pero no por ello se nos da como un lugar vacío, un cero, contrafigura de la plenitud infinita que cada mónada insinúa (Bueno Martínez 44).
\end{abstract}

Esta aproximación resulta muy sugerente con respecto a lo que debiera ser la causa primera del espacio, en tanto que el punto es inextenso - como la mónada-, no tiene partes y es indivisible. Sin embargo, Leibniz propone que una mónada debe tener ciertas cualidades, porque desprovista de ellas, no podrían distinguirse unas de otras. De hecho, propone que cada mónada debe ser distinta a cualquiera "pues no se dan en la naturaleza dos seres que sean perfectamente el uno como el otro, y en donde no sea posible hallar una diferencia interna o fundada en una denominación intrínseca" (Leibniz 77-9). Además, la mónada goza de un cambio continuo que solo puede ser interno, pues ninguna causa exterior puede influirla. Esto significa que el punto podría no ser la mónada de la geometría y menos, el germen del espacio, al no cumplir con tales cualidades.

Entonces, si no es el punto ¿qué es? ¿Cuál es ese primer espacio base de todos los demás? ¿Aquello que se multiplica y en su reproducción crea espacialidades y 
nuevas arquitecturas? Propongo al pliegue. Ya se había mencionado anteriormente que el pliegue puede ser pensado como un evento que da paso al espacio, es decir, la inflexión que afecta la superficie infinita.

Cuando Leibniz habla de la mónada también habla de dos planos que la constituyen, al igual que el real y el ideal constituyen el mundo. Por un lado, está el monádico, punto de vista interno de la mónada, y por el otro, el monadológico que está fuera de ella. Sin embargo, ambos son parte de lo mismo: dos aspectos de una misma cosa (Luna 80-1). Ahora, la relación entre los dos planos se encuentra determinada por la aparición de un pliegue, si se quiere, es el pliegue de estos planos el que origina la mónada: "podemos localizar dos planos concretos, monádico y monadológico, cuyo pliegue dará por resultado la inflexión llamada mónada. Cabe decirlo de otra manera, la inflexión llamada mónada determina un pliegue entre lo monádico y lo monadológico" (Luna 102).

Por lo tanto, el fenómeno del espacio se da en la aparición del pliegue. Si ya no hablamos más de los planos de la mónada, sino de ese plano infinito y flexible que configura el espacio arquitectónico, solamente puede ser el pliegue el que defina sus accidentes. Es más, en los planos de la mónada, el pliegue no solo los diferencia, sino media su relación, tanto como en el espacio media la relación de los existentes. El encuentro de los planos en el espacio está resuelto por el pliegue, es este el que resuelve sus diferencias y los singulariza, es esa línea del pliegue la que caracteriza el espacio y lo configura.

Además de Mónada, el pliegue también aparece, en palabras de Serres, como clinamen. Volvamos al principio del texto. Este filósofo afirma que el pliegue es el átomo de la forma, su elemento, su clinamen que, por definición, se entiende de diversas maneras a lo largo del texto El nacimiento de la física en el texto de Lucrecio: el inicio de la turbulencia, una pequeña desviación, el operador mínimo de la transformación, una experiencia, un transporte; pero, además de estas condiciones formales, también lo propone como el paso de la teoría a la práctica.

En la introducción de la versión inglesa de su texto Hermes. Literature, Science, Philosophy se hace una referencia a esta definición: The clinamen is the operator that marks the passage from the theoretical to the practical: it is the birth of existence. Things come into existence due to the tiny deviations of atoms from laminar flow, the ecart a l'equilibre (xvi) $)^{3}$.

Esta postura resulta fundamentalmente poderosa para lo que quiere probarse no solo en este texto sino en las investigaciones que lo preceden y que seguramente continuaran. Es justamente el pliegue la herramienta que facilita el diálogo entre la teoría y la práctica, en tanto origina el espacio, como se mencionó anteriormente a

3 El clinamen es el operador que marca el paso entre lo teórico y lo práctico: es el nacimiento de la existencia. Las cosas llegan a existir debido a las pequeñas variaciones de átomos de flujo laminar, la desviación del equilibrio [traducido por la autora]. 
FIGURA 3

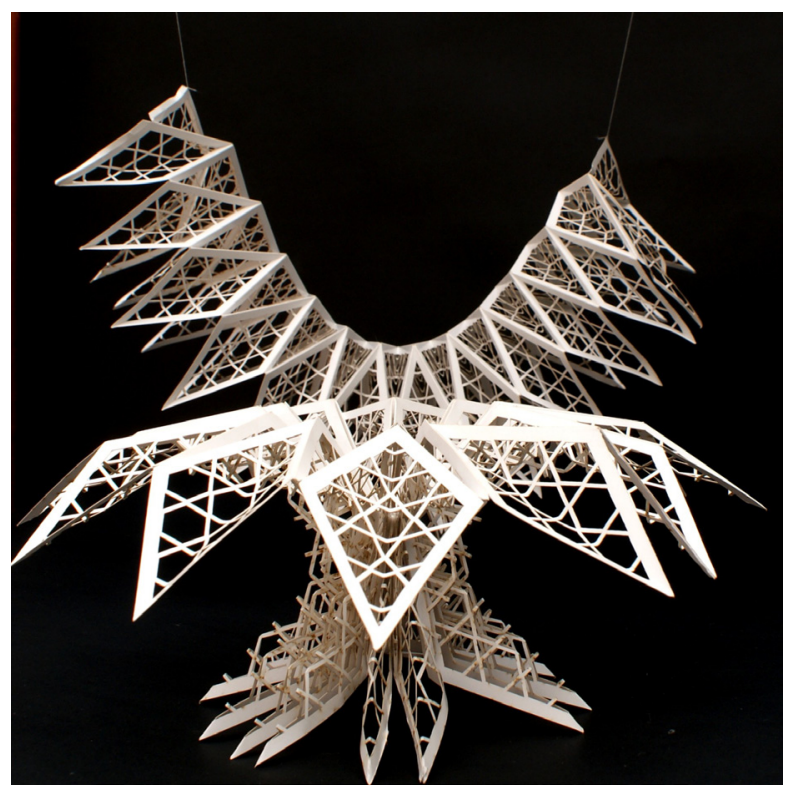

Modelo 6 de 13. Papel común, 120 gr.

\section{FIGURA 4}

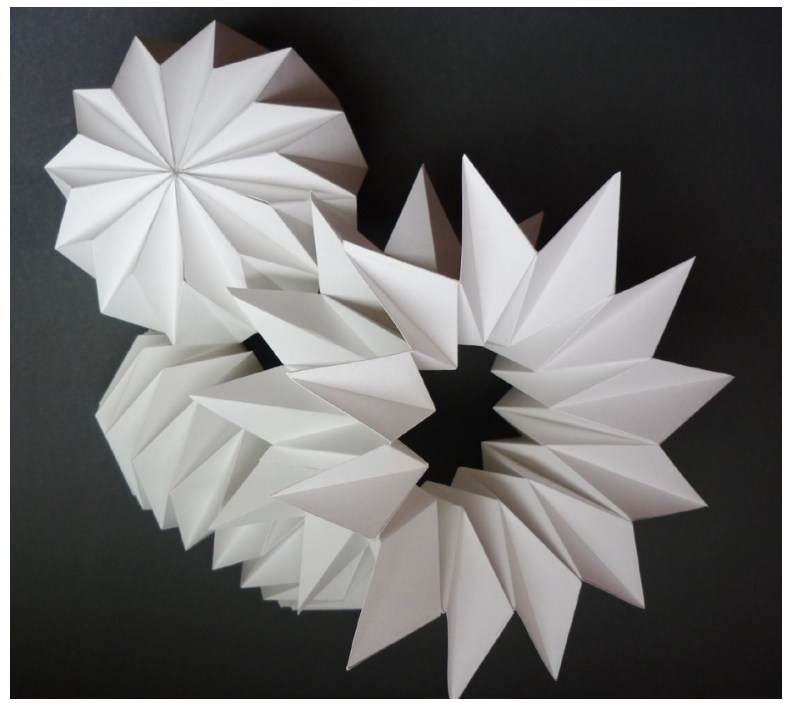

Tessellated Filligree. Presentada en Bridges Enschede, 2013. Cartulina, $120 \mathrm{gr}$. cortada a laser.

propósito de las figuras 3 y 4, las que proponen el pliegue como el evento que turba la superficie y da inicio a una diversidad de emergencias arquitectónicas. Cerca de veinticinco productos formales se han desarrollado hasta ahora, bajo condiciones geométricas y matemáticas, los cuales han expuesto nuevas formas de entender el 
espacio en su plasticidad ${ }^{4} \mathrm{y}$, como se esperaba, evidenciado comportamientos que adquieren forma en el desarrollo teórico. De este modo, el pliegue da inicio a una turbulencia que más adelante se vuelve arquitectura en tanto turba la superficie y permite que se configure.

Pero, ¿si el clinamen es entendido como la suma de varios? En ese caso el pliegue en sí mismo no es suficiente, necesita de otros que lo validen, necesita una desviación, una vez más, un clinamen, o en palabras de Jean Luc Nancy "hace falta una inclinación del uno hacia el otro, del uno por el otro o del uno al otro" (15). Si para Nancy la comunidad es el clinamen del individuo, aquí el hecho arquitectónico será del pliegue.

En resumen, todo el espacio arquitectónico, construido, pensado y por pensar, reposa sobre una superficie infinita, flexible e inagotable, y cada intervención en ella puede leerse como pliegue e inflexión. Además, dicho pliegue garantiza la aparición de singularidades que, aun cuando pertenecen a un sistema ordenado y homogéneo, al vincularse a una misma naturaleza, no son leídos como iguales y promueven atributos singulares en dicha superficie. Además, dicho espacio es fundamentalmente continuo al estar compuesto de una vasta diversidad de pliegues. Desde tal condición el espacio arquitectónico se establece como un cuerpo plástico modificable, pero, sobre todo, el espacio arquitectónico existirá en tanto existan pliegues que permitan su aparición.

Queda todavía mucho por pensar sobre este paso transdisciplinar, no solo de Leibniz a la arquitectura sino de todo aquello que se considere como detonante y origen. Por lo que se espera, y con ansias, volver a este lugar de creación teórica y ver de qué forma va tomando maduración.

\section{Referencias}

Augé, Marc. Los no lugares. Espacios del anonimato. Barcelona: Gedisa, 1992. Impreso. Bueno Martínez, Gustavo. "Introducción a la monadología de Leibniz". Monadología. Gottfried Leibniz. Oviedo: Pentalfa, 1981. 11-47. Impreso.

Cáceres Hernández, José Juan. Conceptos básicos de estadistica para Ciencias Sociales. Madrid: Delta, 2007. Impreso.

Centelles Portella, Josep. El buen gobierno de la ciudad: estrategias urbanas y política relacional. La Paz: Plural, 2006. Impreso.

Centro Andaluz de Arte Contemporáneo. "Junta de Andalucía”. Hoja de Sala "Exposición Equipo 57”. 19 Dic. 2007. Web. 15 Feb. 2011.

Colodro, Max. Reflexiones sobre el caos. Santiago: Universitaria, 2002. Impreso.

Deleuze, Gilles. "De la curva al individuo. Inflexión, punto de vista y mónada” El Leibniz de Deleuze. Buenos Aires: Cactus, 2006. 139-171. Impreso.

4 Gran parte de estas propuestas las desarrollo en el texto El espacio continuo: Aproximaciones del libro Estética y sistemas abiertos. Procesos de no equilibrio entre el arte, la ciencia y la ciudad (2013, 215-34). 
Granados, Maritza. “¿Ocupar o habitar? Aproximación al fenómeno actual”. Arte, Individuo y Sociedad 25.5 (2013): 376-391. Impreso.

Leibniz, Gottfried Wilhelm. Monadología. Oviedo: Pentalfa, 1981. Impreso.

Luna, Manuel. La ley de la continuidad en G. W. Leibniz. Tesis Doctoral, Facultad de Filosofía, Universidad de Sevilla, 1994. Impreso.

Monlau, Pedro Felipe. Diccionario etimológico de la lengua castellana. Madrid: Imprenta y Esterotipia de M. Rivadeneyra, 1856. Impreso.

Nancy, Jean Luc. La comunidad inoperante. Santiago de Chile: ARCIs/Lom, 2000. Impreso.

Oleastro, Joaquín. “Oritectura. Estudio sobre el origami y la arquitectura”. Ed. Ezequiel Usón. Dimensiones de la sostenibilidad. Barcelona: UPC, 2004. 85-116. Impreso.

Pérgolis, Juan Carlos. La ciudad deseada. Buenos Aires: Nobuko, 2005. Impreso.

Serres, Michel. El nacimiento de la física en el texto de Lucrecio. Valencia: Pre-Textos, 1977. Impreso.

---. Hermes. Literature, science, philosophy. Londres: John Hopkins University Press, 1982. Impreso.

Ureña, Gabriel. Las vanguardias artísticas en la postguerra española, 1940-1959. Madrid: Istmo, 1982. Impreso.

Recibido: 19 agosto 2016

Aceptado: 28 agosto 2016 